\title{
Heterosis in Early Maize Ear Inflorescence Development: A Genome-Wide Transcription Analysis for Two Maize Inbred Lines and Their Hybrid
}

Haiping Ding ${ }^{1, \dagger}$, Cheng Qin ${ }^{1,2, \dagger}$, Xirong Luo ${ }^{2}$, Lujiang Li ${ }^{1}$, Zhe Chen ${ }^{1}$, Hongjun Liu ${ }^{1}$, Jian Gao ${ }^{1}$, Haijian Lin ${ }^{1}$, Yaou Shen ${ }^{1}$, Maojun Zhao ${ }^{3}$, Thomas Lübberstedt ${ }^{4}$, Zhiming Zhang ${ }^{1, *}$ and Guangtang Pan ${ }^{1}, *$

1 Maize Research Institute of Sichuan Agricultural University/Key Laboratory of Biology and Genetic Improvement of Maize in Southwest Region, Ministry of Agriculture, Chengdu 611130, China; E-Mails: dinghp@sicau.edu.cn (H.D.); cheng.qin.sicau@gmail.com; (C.Q.);

lilujiang2014@gmail.com (L.L.); chenzhe1024@gmail.com (Z.C.); 1hj20305@gmail.com (H.L.); gaojian8888@gmail.com (J.G.); linhj521@gmail.com (H.L.); shenyaou@gmail.com (Y.S.)

2 Zunyi Academy of Agricultural Sciences, Zunyi 563102, China;

E-Mail: luoxirong2014@gmail.com

3 Life Science College, Sichuan Agricultural University, Ya'an 625014, China;

E-Mail: toxic.xc@gmail.com

4 Department of Agronomy, Iowa State University, Ames, IA 50011, USA;

E-Mail: thomas1@iastate.edu

$\dagger$ These authors contributed equally to this work.

* Authors to whom correspondence should be addressed;

E-Mails: zzmmaize@gmail.com (Z.Z.); pangt@sicau.edu.cn (G.P.);

Tel.: +86-28-8629-0917 (G.P.); Fax: +86-28-8629-0916 (G.P.).

Received: 10 May 2014; in revised form: 1 July 2014 / Accepted: 2 July 2014 /

Published: 11 August 2014

Abstract: Heterosis, or hybrid vigor, contributes to superior agronomic performance of
hybrids compared to their inbred parents. Despite its importance, little is known about the
genetic and molecular basis of heterosis. Early maize ear inflorescences formation affects
grain yield, and are thus an excellent model for molecular mechanisms involved in
heterosis. To determine the parental contributions and their regulation during maize
ear-development-genesis, we analyzed genome-wide digital gene expression profiles in
two maize elite inbred lines (B73 and Mo17) and their $\mathrm{F}_{1}$ hybrid using deep sequencing 
technology. Our analysis revealed 17,128 genes expressed in these three genotypes and 22,789 genes expressed collectively in the present study. Approximately $38 \%$ of the genes were differentially expressed in early maize ear inflorescences from heterotic cross, including many transcription factor genes and some presence/absence variations (PAVs) genes, and exhibited multiple modes of gene action. These different genes showing differential expression patterns were mainly enriched in five cellular component categories (organelle, cell, cell part, organelle part and macromolecular complex), five molecular function categories (structural molecule activity, binding, transporter activity, nucleic acid binding transcription factor activity and catalytic activity), and eight biological process categories (cellular process, metabolic process, biological regulation, regulation of biological process, establishment of localization, cellular component organization or biogenesis, response to stimulus and localization). Additionally, a significant number of genes were expressed in only one inbred line or absent in both inbred lines. Comparison of the differences of modes of gene action between previous studies and the present study revealed only a small number of different genes had the same modes of gene action in both maize seedlings and ear inflorescences. This might be an indication that in different tissues or developmental stages, different global expression patterns prevail, which might nevertheless be related to heterosis. Our results support the hypotheses that multiple molecular mechanisms (dominance and overdominance modes) contribute to heterosis.

Keywords: maize (Zea mays L.); heterosis; DGE (digital gene expression); differentially expressed genes; multiple molecular mechanisms

\section{Introduction}

Heterosis, or hybrid vigor, refers to the phenomenon in which progeny of two inbred lines (hybrids) exhibit enhanced agronomic performance such as biomass production, growth rate, fertility, and disease resistance relative to both parents [1]. Heterosis has been extensively used in agriculture, especially in the breeding of maize and rice. For example, it is estimated that approximately $95 \%$ of the United States maize acreage and 55\% of rice acreage in China are planted with hybrids. Furthermore, hybrid maize technology for large-scale production has a yield advantage of $15 \%$ over the elite inbred varieties. Though the concept of heterosis has been introduced over 100 years ago and different genetic models considered [1-3], the genetic and molecular basis of heterosis remains elusive.

Three classical genetic hypotheses to explain heterosis have been proposed: the dominance, overdominance, and epistasis hypotheses. The dominance hypothesis states that deleterious recessive alleles cause inbreeding depression. A cross of two inbred parents will benefit from complementation of these deleterious alleles and will display a superior phenotype [2-4]. The over-dominance hypothesis refers to allelic interactions at one or multiple heterozygous genes resulting in superior trait expression compared to the better parent $[1,5]$. Both hypotheses may be insufficient to explain the molecular mechanism for heterosis [6]. Epistasis hypothesis refers to interactions of alleles at different loci from two parents in $\mathrm{F}_{1}$ hybrids, leading to heterosis $[7,8]$. 
Recently, genetic and molecular studies provided experimental support for above-mentioned hypotheses. Using molecular markers in segregating populations, quantitative trait locus (QTL) mapping studies provided support for the dominance [9], over-dominance [10], and epistasis [7,8] models. With the advent of genomic methods to assay genome-wide patterns of gene expression, recent studies indifferent tissues and developmental stages of model and crop plants (such as maize, rice, wheat, Populus, and Arabidopsis) have determined the roles of different gene expression [11-32], small RNAs [33-36], and epigenetic regulation [37,38], including circadian-mediated metabolic pathways [39], in heterosis. For instance, additive gene expression was prevalent in some studies of maize $[14,26,32]$, and there were varying numbers of genes that exhibited a non-additive behavior in other studies [12,17-27,29,30,35].

Maize immature ear inflorescences show heterosis in ear architectural traits [40], and significant positive correlations between grain yield and ear architectural traits, such as ear length, kernel row number, number of kernels per row, kernel number density, and cob diameter have been reported [40], which suggests that each of these components contributes to greater yields. A thorough knowledge of the genes affecting the various components and their interactions will facilitate our understanding of the molecular basis of heterosis of grain yield. In this study, we applied a highly effective approach of high throughout deep sequencing to identify genes, which are highly expressed in maize elite inbred lines of B73 and Mo17, and their $F_{1}$ hybrid $(B 73 \times$ Mo17) at an early stage of ear inflorescences development. We provide first molecular evidence that regulatory mechanisms underlying the phenomenon of heterosis are very early active in maize ear development. Furthermore, we also found that differentially expressed genes between hybrids and their parents can be involved in certain regulatory networks, which suggested that complicated gene networks might be underlying heterosis. Results of the present study might help promote further understanding of mechanisms underlying heterosis.

\section{Results}

\subsection{Statistics and Analysis of Library Sequencing}

Here, we sequenced three ear digital gene expression (DGE) libraries from two inbred parents $\left(\mathrm{B} 73\right.$ and Mo17) and their $\mathrm{F}_{1}$ hybrid $(\mathrm{B} 73 \times \mathrm{Mo17})$ using massively parallel sequencing on the Illumina platform at BGI-Shenzhen, China (Tables 1 and S1). A total of approximately 4.2 million raw tags per library with 259,890 distinct tag sequences were identified. After data-processing steps (see Materials and Methods), the total number of filtered, high-quality clean tags was almost the same in three libraries. Furthermore, the $F_{1}$ library had the highest number of distinct tags $(259,282)$, followed by the B73 $(242,184)$, and Mo17 $(239,963)$ libraries (Table 1 and S1). These distinct tags and their genomic frequency as well as the raw data were deposited in NCBI Sequence Read Archive (SRA) database with the accession number (PRJNA248701). Copy numbers of most of the distinct tags (over $77 \%$ ) ranged from 1-5. However, a small number of distinct tags (less than $3.29 \%$ ) with a frequency higher than 100 make up over 62\% of all clean tags in all three libraries (Figure S1).

When sequencing depths reach 1 million total tags, the number of novel distinct tags discovered dropped dramatically in all three libraries (Figure S2A). From that point, increasing sequence depth results in a slow and stable accumulation of new distinct tags indicating that sequencing has reached 
saturation. Moreover, as shown in Figure 2B, when the total tag number in B73 reached 1 million, the increase of identified genes started to level out, and stabilized when the number of tags reached 3 million. Next, the level of gene expression was determined by calculating the number of unambiguous clean tags for each gene and then normalized to the number of transcripts per million tags (TPM). The Mo17 and $F_{1}$ data showed a similar trend (Figure S2). This suggests that only few more distinct genes would be identified when the total clean tag number reached a certain value.

Table 1. Summary statistics from mapping digital gene expression (DGE) sequence tags to the maize B73 reference genome.

\begin{tabular}{lllll}
\hline Class & Summary & $\mathbf{F}_{1}$ & $\mathbf{B 7 3}$ & Mo17 \\
\hline \multirow{2}{*}{ Raw Data } & Total & $4,200,000$ & $4,200,000$ & $4,200,000$ \\
& Distinct Tags & 272,402 & 254,882 & 252,386 \\
\hline \multirow{2}{*}{ Clean Tags } & Total number & $4,176,622$ & $4,176,825$ & $4,176,752$ \\
& Distinct Tags number & 259,282 & 242,184 & 239,963 \\
\hline \multirow{4}{*}{ All Tags Mapping to Genome } & Total number & $2,766,685$ & $2,979,615$ & $3,127,520$ \\
& Total \% of clean tags & $66.24 \%$ & $71.34 \%$ & $74.88 \%$ \\
& Distinct Tags number & 134,449 & 134,378 & 141,476 \\
& Distinct Tags \% of clean tags & $51.85 \%$ & $55.49 \%$ & $58.96 \%$ \\
\hline \multirow{4}{*}{ Unambiguous Tags Mapping to Genomer } & Total number & $2,452,293$ & $2,637,419$ & $2,804,389$ \\
& Total \% of clean tags & $58.71 \%$ & $63.14 \%$ & $67.14 \%$ \\
& Distinct Tags number & 119,316 & 118,719 & 125,467 \\
& Distinct Tags \% of clean tags & $46.02 \%$ & $49.02 \%$ & $52.29 \%$ \\
\hline \multirow{2}{*}{ All Tags Mapping to Genes } & Number & 24,629 & 24,078 & 24,198 \\
& \% of ref. genes & $75.69 \%$ & $74 \%$ & $74.36 \%$ \\
\hline \multirow{2}{*}{ Unambiguous Tags Mapping to Genes } & Number & 21,372 & 20,784 & 20,938 \\
& \% of ref. genes & $65.68 \%$ & $63.87 \%$ & $64.35 \%$ \\
\hline \multirow{3}{*}{ Unknown Tags } & Total number & 894,503 & 721,786 & 561,476 \\
& Total \% of clean tags & $21.42 \%$ & $17.28 \%$ & $13.44 \%$ \\
& Distinct Tags number & 85,152 & 70,700 & 61,016 \\
& Distinct Tags \% of clean tags & $32.84 \%$ & $29.19 \%$ & $25.43 \%$ \\
\hline
\end{tabular}

\subsection{Mapping Tags to the Maize Reference Genome}

We used SOAP2 software [41] to map all distinct tags to the maize reference genome (B73 RefGen_v2) [42]. Mapping results showed that 51.85\%, 55.49\% and 58.96\%, respectively, of distinct clean tags mapped to the reference database (sense or anti-sense), and 46.02\%, 49.02\%, and 52.29\%, respectively, of the distinct clean tags mapped unambiguously to the reference genes (Table 1 and S1). Out these, we identified 20,784-21,372 genes expressed in the three genotype comparison (Table S2), our analysis revealed 17,128 genes expressed in all samples and 22,789 genes expressed collectively in the present study (Table S3). In total, 32.84\%, 29.19\%, and $25.43 \%$ of all distinct clean tags for $\mathrm{F}_{1}$, B73, and Mo17 data sets, respectively, did not map to the reference maize genome sequence or associated transcripts (Tables 1 and S1). These non-mapped tags most likely represent regions where the maize reference sequence is incomplete [43] or there are differential mRNA processing events for most maize genes, such as alternative splicing [44]. Only $0.03 \%$ of non-mapped tags matched 
maize chloroplast or mitochondrial genome sequences (Table S1). Because Solexa sequencing can distinguish transcripts originating from both DNA strands, we found evidence for bidirectional transcription in 14,012-14,420 of all detectable overlapping genes and 1031-1119 antisense-stand specific transcripts based on the strand-specific nature of the sequenced tags (Table S2). By comparison, the ratio of sense to antisense strand of the transcripts was approximately 1.3:1 for all libraries. As summarized in Figure S3 and Table S3, most expressed genes (approx. 19,965) are represented in fewer than one hundred copies and only a small proportion of genes are highly expressed (Table S3). We map the clean tags, which cannot be mapped to mRNA, mitochondria and chloroplasts, to the whole genome, providing start positions that could be uniquely mapped by those tags (Table S4).

\subsection{Different Gene Expression Analysis}

A total of 32,973 significantly changed tags entities were detected among three genotypes (see Materials and Methods) (Figure S4A,C,E and Table S5). Then, the processed DGE data were used to determine different gene expression between inbred parents or between parental lines and their $\mathrm{F}_{1}$ hybrid and we identified 8621 out of 22,789 genes that were differentially expressed among B73, Mo17, and their hybrid, representing 38\% of the ear digital gene expression (Figure 1A). Among them, 3401 (3344) and 2226 (2189) genes were significantly up-regulated and down-regulated, respectively, in the $F_{1}$ compared to B73/Mo17 (Figure 1B, Figure S4B,D and Table S6). The comparison between parental line libraries also revealed significant variation in expression. A total of 3883 genes, including 1896 up-regulated and 1987 down-regulated genes, were identified in B73 compared to Mo17 (Figure 1B, Figure S4F and Table S6).

Figure 1. Differentially expressed genes in the maize heterotic cross. Venn diagram (a) and statistical analysis (b) of differentially expressed genes among inbred parents and their $\mathrm{F}_{1}$ hybrid.

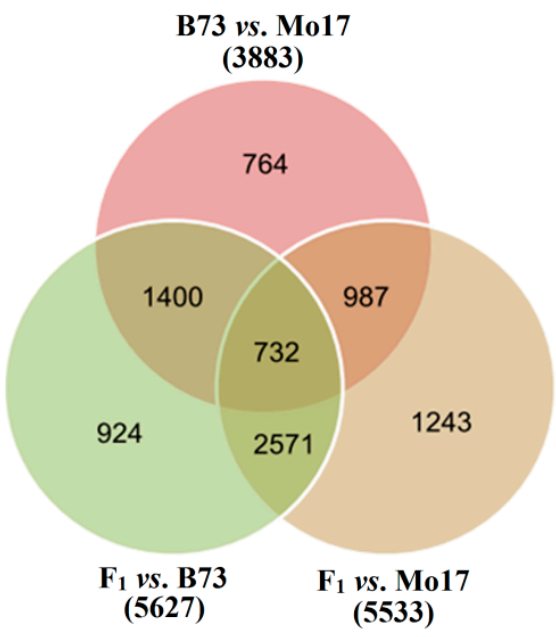

(a)

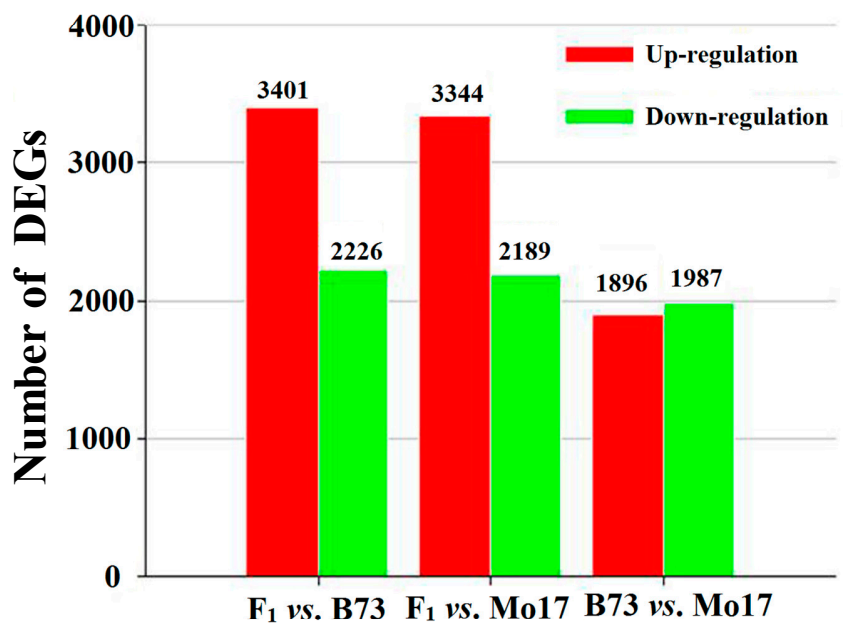

(b)

We further investigated the mode of gene action for these different genes (Table S6). 8.9\% (767 of 8621) exhibited an expression pattern that was not distinguishable from additivity, while the other 91.1\% (7854 of 8621) genes showed non-additive expression patterns (Figure 2A). The non-additive 
differentially expressed genes from the cross were further classified into four distinct classes: high-parent dominance (1984), low-parent dominance (2559), over-dominance (1085), and under-dominance (1963) (Figure 2B and Table 2). A sample of 30 differentially expressed genes was randomly selected for validation by qRT-PCR. The trends in the expression of these genes detected by DGE were consistent (29 genes) or partially (1 gene) consistent with those determined in qRT-PCR analyses (Figure 3 ). These findings are consistent with a recent study in maize [31] or rice [16] that supports the involvement of multiple modes (dominance and overdominance) of gene action in association with heterosis.

Figure 2. Functional categories of differentially expressed genes. Overall differentially expressed genes (a) and non-additive expressed genes (b) from B73 $\times$ Mo17 cross.

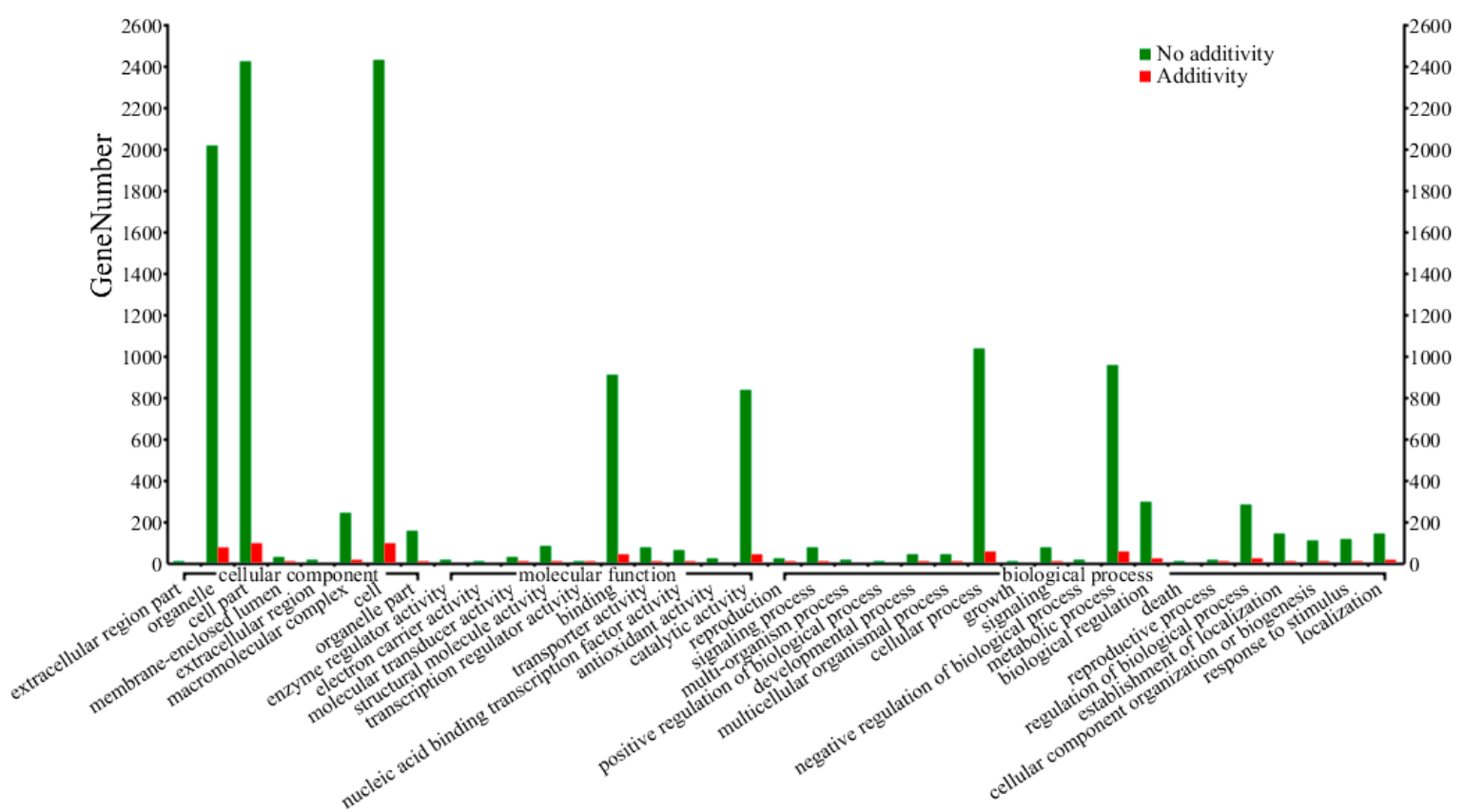

(a)

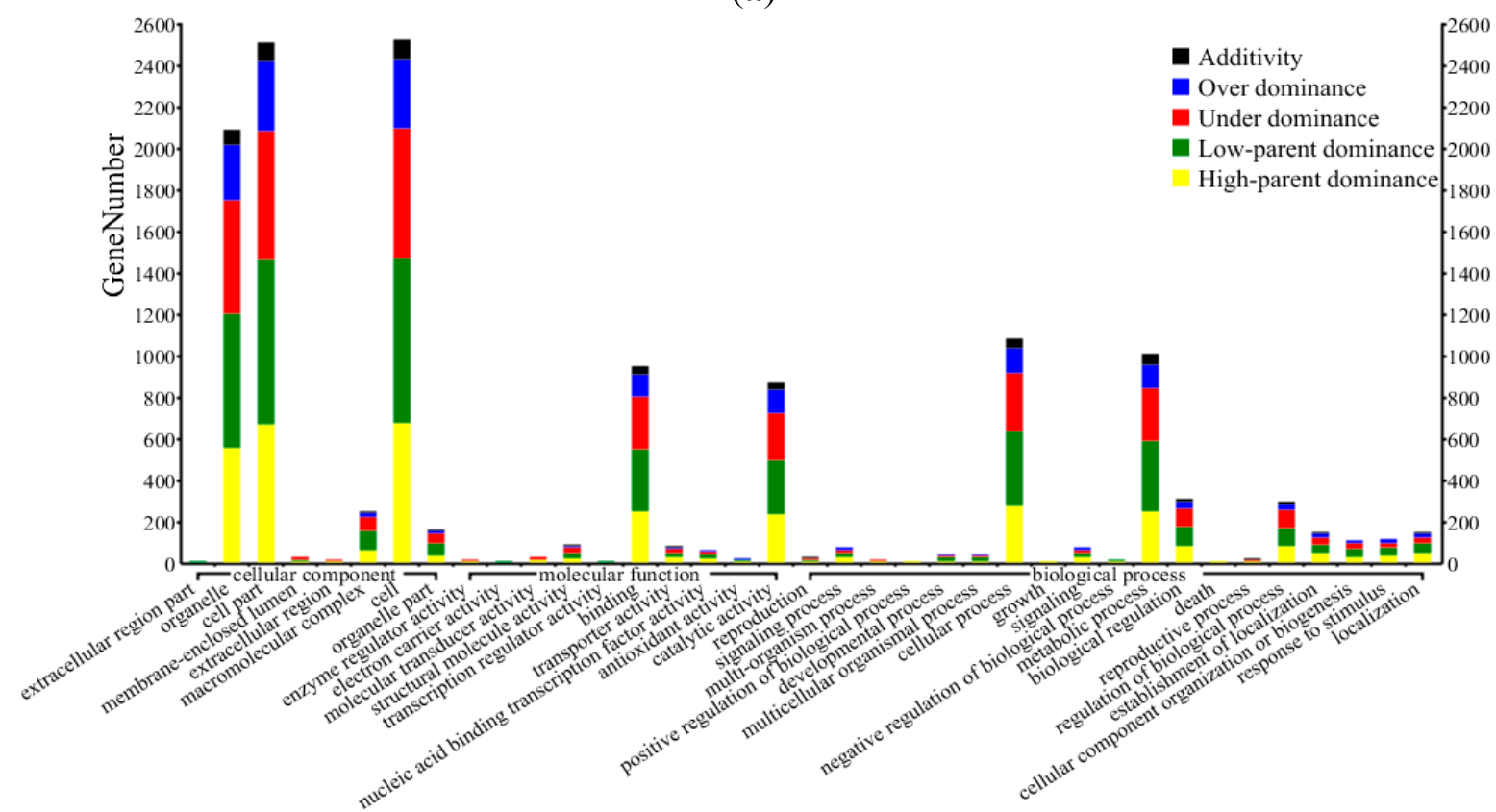

(b) 
Table 2. Statistical analysis of differentially expressed genes (FDR $\leq 0.001$ and absolute value of $\log _{2}$ Ratio $\geq 1$ ).

\begin{tabular}{cc}
\hline Hybrid Cross & $\mathrm{B} 73 \times \mathrm{Mo17}$ \\
\hline Total & 8621 \\
Additivity & 767 \\
Non Additivity & 7854 \\
High-Parent Dominance & 1984 \\
Low-Parent Dominance & 2559 \\
Over Dominance & 1085 \\
Under Dominance & 1963 \\
Other & 263 \\
\hline
\end{tabular}

$\mathrm{F}_{1}$ represents the hybrid line; $\mathrm{P}$, paternal line, $\mathrm{B} 73$; and $\mathrm{M}$, maternal line, Mo17. Additivity, $\mathrm{F}_{1} \approx 1 / 2(\mathrm{P}+\mathrm{M})$; non-additivity, $\mathrm{F}_{1}>1 / 2(\mathrm{P}+\mathrm{M})$ or $\mathrm{F}_{1}<1 / 2(\mathrm{P}+\mathrm{M})$. High-parent dominance (HPD), $\mathrm{F}_{1} \approx \mathrm{P}>\mathrm{M}$ or $\mathrm{F}_{1} \approx \mathrm{M}<\mathrm{P}$; low-parent dominance (LPD), $\mathrm{F}_{1} \approx \mathrm{P}<\mathrm{M}$ or $\mathrm{F}_{1} \approx \mathrm{M}<\mathrm{P}$; over-dominance (ODO), $\mathrm{F}_{1}>\mathrm{P}$ and $\mathrm{F}_{1}>\mathrm{M}$; under-dominance (UDO), $\mathrm{F}_{1}<\mathrm{P}$ and $\mathrm{F}_{1}<\mathrm{M}$.

Figure 3. Expression of 12 differentially expressed gene from arginine and proline metabolism, pyruvate metabolism, and purine metabolism in the maize heterotic cross. The expression pattern of 12 genes detected by digital gene expression platform (a) and quantitative Real-Time PCR (qRT-PCR) (b). The black, red, and green bars in graph b depicted the stem-loop qRT-PCR relative expression level \pm standard error of three replicates for each gene in B73, Mo17 and their hybrid.

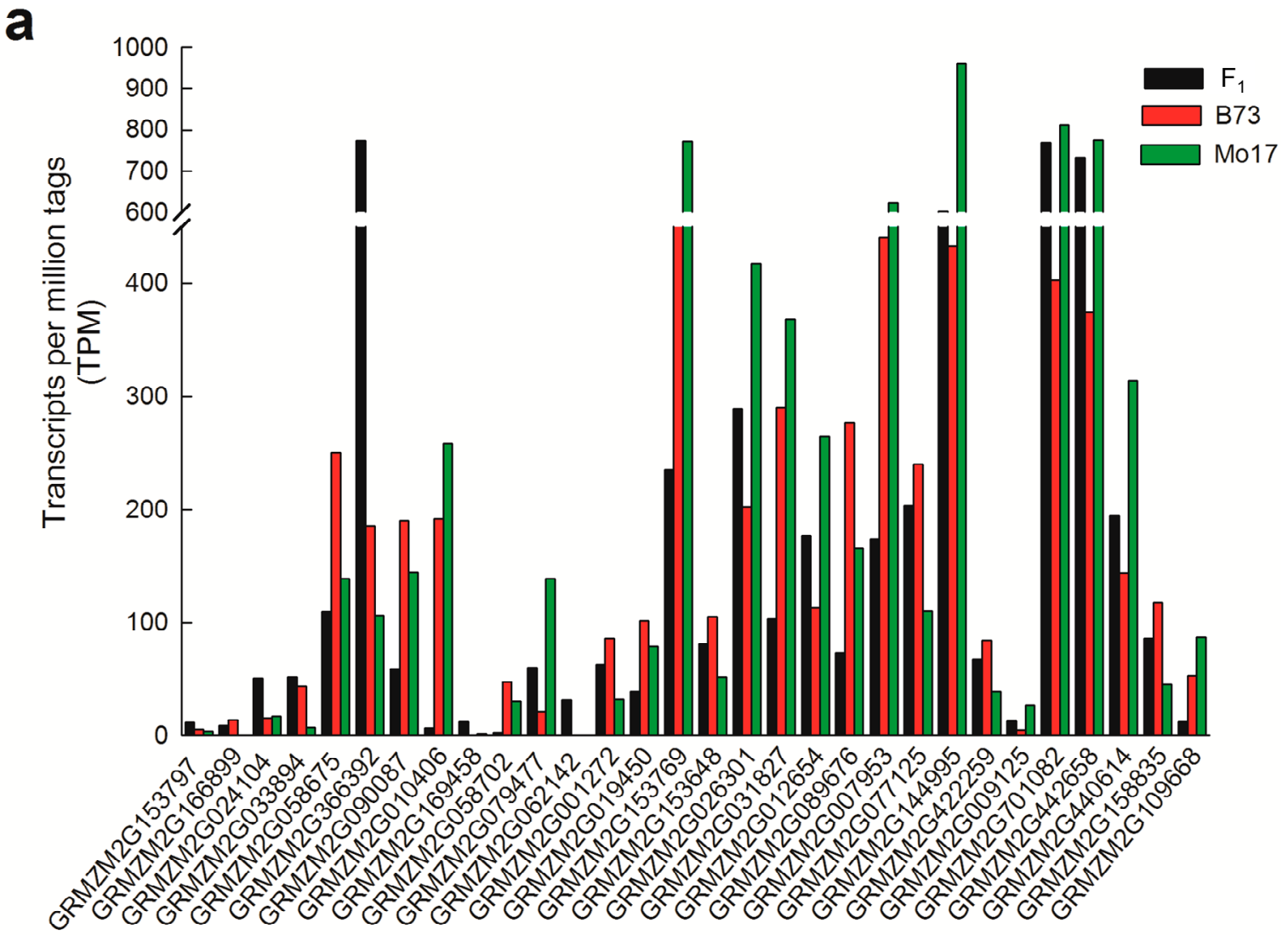


Figure 3. Cont.

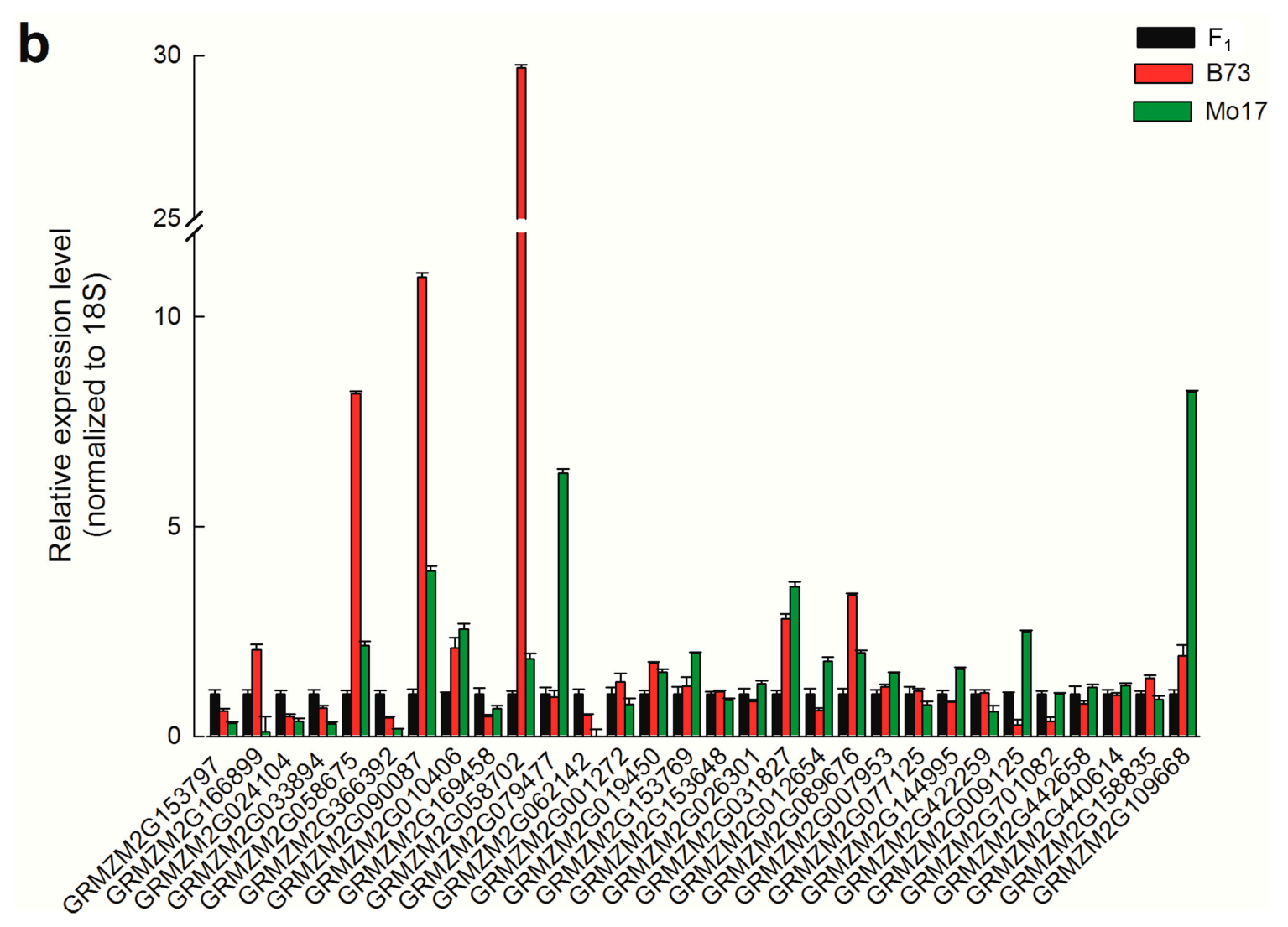

\subsection{Functional Enrichment Analysis for Differentially Expressed Genes Using Gene Ontology (GO)}

To gain a better understanding of the functional roles of different genes between inbred parents or between parental lines and their $\mathrm{F}_{1}$ hybrid, we looked for gene enrichment regarding the Gene Ontology (GO) cellular component, molecular function and biological process categories [44,45]. Functional-annotations from the maize genome (http://maizesequence.org) were used for functional classification of the 8621 different genes and performed by the official web-based tools for searching and browsing the Gene Ontology database (AmiGO) (http://www.geneontology.org/) [45] (Figure 2 and Table 3). We found that different genes showing differential expression patterns were mainly enriched in five cellular component categories (organelle, cell, cell part, organelle part and macromolecular complex), five molecular function categories (structural molecule activity, binding, transporter activity, nucleic acid binding transcription factor activity and catalytic activity), and eight biological process categories (cellular process, metabolic process, biological regulation, regulation of biological process, establishment of localization, cellular component organization or biogenesis, response to stimulus and localization) (Table 3). We further classified different genes in more detail based on Gene Ontology (GO) by AmiGO [45] (Tables S7-S9). These different genes in GO annotation analysis of cellular component showed significant enrichment in the following components: non-membrane-bound organelle $\left(6.40 \times 10^{-4}\right)$, intracellular non-membrane-bound organelle $\left(6.40 \times 10^{-4}\right)$, nucleus $\left(2.56 \times 10^{-3}\right)$, ribosome $\left(3.34 \times 10^{-2}\right)$, and macromolecular complex $\left(4.27 \times 10^{-2}\right)$. In the GO biological process enrichment analysis, there are five significant GO terms (FDR corrected $p$-value $<0.05$ ). Eighty percent were macromolecular synthesis-related, such as 
cellular protein metabolic process, cellular macromolecule metabolic process, protein metabolic process, and macromolecule metabolic process (Table 4).

Table 3. Functional classification of different genes between inbred parents or between parental lines and their $\mathrm{F}_{1}$ hybrid. * indicated the significant enrichment in functional classification.

\begin{tabular}{|c|c|c|c|c|c|c|c|}
\hline Functional Categories & Additivity & Non-Additivity & HPD & LPD & UDO & ODO & Other \\
\hline \multicolumn{8}{|c|}{ Cellular Component } \\
\hline Extracellular Region Part & 1 & 3 & 0 & 2 & 0 & 1 & 0 \\
\hline Organelle * & 181 & 2077 & 544 & 650 & 542 & 271 & 70 \\
\hline Cell Part & 221 & 2499 & 655 & 793 & 624 & 339 & 88 \\
\hline Membrane-Enclosed Lumen & 1 & 24 & 3 & 9 & 10 & 1 & 1 \\
\hline Extracellular Region & 1 & 13 & 1 & 5 & 2 & 5 & 0 \\
\hline Macromolecular Complex * & 16 & 245 & 58 & 89 & 71 & 16 & 11 \\
\hline Cell & 221 & 2510 & 660 & 794 & 628 & 340 & 88 \\
\hline Organelle Part & 16 & 155 & 31 & 57 & 47 & 14 & 6 \\
\hline \multicolumn{8}{|c|}{ Molecular Function } \\
\hline Enzyme Regulator Activity & 3 & 12 & 3 & 2 & 3 & 4 & 0 \\
\hline Electron Carrier Activity & 0 & 3 & 0 & 1 & 2 & 0 & 0 \\
\hline Molecular Transducer Activity & 3 & 24 & 7 & 5 & 9 & 2 & 1 \\
\hline Structural Molecule Activity & 3 & 80 & 17 & 27 & 28 & 4 & 4 \\
\hline Transcription Regulator Activity & 2 & 6 & 0 & 1 & 2 & 2 & 1 \\
\hline Binding & 75 & 940 & 241 & 297 & 258 & 108 & 36 \\
\hline Transporter Activity & 9 & 76 & 25 & 19 & 20 & 9 & 3 \\
\hline Nucleic Acid Binding Transcription Factor Activity & 2 & 58 & 18 & 19 & 15 & 2 & 4 \\
\hline Antioxidant Activity & 0 & 17 & 5 & 3 & 3 & 6 & 0 \\
\hline Catalytic Activity & 67 & 865 & 229 & 263 & 226 & 111 & 36 \\
\hline \multicolumn{8}{|c|}{ Biological Process } \\
\hline Reproduction & 0 & 21 & 3 & 8 & 5 & 3 & 2 \\
\hline Signaling Process & 5 & 70 & 24 & 20 & 16 & 7 & 3 \\
\hline Multi-Organism Process & 0 & 8 & 4 & 2 & 1 & 1 & 0 \\
\hline Positive Regulation of Biological Process & 0 & 3 & 2 & 1 & 0 & 0 & 0 \\
\hline Developmental Process & 2 & 38 & 2 & 21 & 9 & 4 & 2 \\
\hline Multicellular Organismal Process & 2 & 39 & 4 & 20 & 9 & 4 & 2 \\
\hline Cellular Process & 71 & 1079 & 269 & 357 & 281 & 125 & 47 \\
\hline Growth & 0 & 5 & 1 & 2 & 1 & 1 & 0 \\
\hline Signaling & 5 & 70 & 24 & 20 & 16 & 7 & 3 \\
\hline Negative Regulation of Biological Process & 2 & 12 & 2 & 6 & 3 & 1 & 0 \\
\hline Metabolic Process & 70 & 999 & 242 & 338 & 253 & 118 & 48 \\
\hline Biological Regulation & 18 & 303 & 77 & 94 & 88 & 28 & 16 \\
\hline Death & 2 & 6 & 2 & 1 & 2 & 1 & 0 \\
\hline Reproductive Process & 0 & 15 & 1 & 5 & 5 & 2 & 2 \\
\hline Regulation of Biological Process & 17 & 289 & 76 & 88 & 84 & 26 & 15 \\
\hline Establishment of Localization & 15 & 143 & 41 & 44 & 33 & 19 & 6 \\
\hline Cellular Component Organization or Biogenesis & 10 & 106 & 24 & 38 & 29 & 10 & 5 \\
\hline Response to Stimulus & 4 & 112 & 33 & 37 & 22 & 19 & 1 \\
\hline Localization & 16 & 145 & 41 & 45 & 33 & 19 & 7 \\
\hline
\end{tabular}


Table 4. Significant GO terms of DGs in the gene ontology (GO) annotation analysis of cellular component and biological process. ${ }^{a} p$-values calculated using a hypergeometric test-determines if the number of times that a GO term appears in the cluster is significant, relative to its occurrence in the genome.

\begin{tabular}{lll}
\hline GO Term & Definition & FDR Corrected $\boldsymbol{p}$-Value \\
\hline Cellular Component & & \\
\hline GO:0043228 & Non-membrane-bounded organelle & $6.40 \times 10^{-4}$ \\
GO:0043232 & Intracellular non-membrane-bounded organelle & $6.40 \times 10^{-4}$ \\
GO:0005634 & Nucleus & $2.56 \times 10^{-3}$ \\
GO:0005840 & Ribosome & $3.34 \times 10^{-2}$ \\
GO:0032991 & Macromolecular complex & $4.27 \times 10^{-2}$ \\
\hline Biological Process & & \\
\hline GO:0044267 & Cellular protein metabolic process & $4.22 \times 10^{-3}$ \\
GO:0044260 & Cellular macromolecule metabolic process & $8.84 \times 10^{-3}$ \\
GO:0019538 & Protein metabolic process & $3.30 \times 10^{-2}$ \\
GO:0009987 & Cellular process & $3.37 \times 10^{-2}$ \\
GO:0043170 & Macromolecule metabolic process & $4.59 \times 10^{-2}$ \\
\hline
\end{tabular}

\subsection{The Expression Patterns of Biological Macromolecular Synthesis-Related Genes in the} B73 $\times$ Mo17 Hybrid

To investigate pathways in which different genes were involved and enriched, pathway analysis was performed using the KOBAS 2.0 web tool [46]. 2935 out of 5627 different genes were involved in 122 pathways between $F_{1}$ and B73, 2877 out of 5533 different genes were involved in 121 pathways between $\mathrm{F}_{1}$ and Mo17 (Table S10), Among them, the majority were present in ribosome, spliceosome and various metabolic pathways (such as arginine and proline metabolism, purine metabolism, glycolysis/gluconeogenesis, and biosynthesis of alkaloids derived from histidine and purine, etc.) (Table S10). $q$-value estimates [47] revealed only four pathways showing significance $(q<0.05)$ : ribosome $\left(q=1.58 \times 10^{-3}\right)$, arginine and proline metabolism $\left(q=1.58 \times 10^{-3}\right)$, spliceosome $\left(q=1.63 \times 10^{-2}\right)$ and pyruvate metabolism $\left(q=3.80 \times 10^{-2}\right)$ (Tables 5 and S11), and the first two pathways showed extreme significance $(q<0.01)$. Of the top 10 differentially expressed genes enriched in pathways between parental lines and their $F_{1}$ hybrid, it should be noted that the purine metabolism pathway was the third largest pathway, following the ribosome pathway, although it does not reach the significance level (Tables 5 and S11).

Table 5. Top 10 pathways differentially expressed genes enriched in between parental lines and their $\mathrm{F}_{1}$ hybrid.

\begin{tabular}{|c|c|c|c|c|c|c|c|}
\hline \multirow{2}{*}{ Pathway $^{\text {a }}$} & \multicolumn{4}{|c|}{ Different Genes with Pathway Annotation (4312) } & \multirow{2}{*}{$p$-Value ${ }^{b}$} & \multirow{2}{*}{$q$-Value ${ }^{\mathrm{c}}$} & \multirow{2}{*}{$\begin{array}{c}\text { Pathway } \\
\text { ID } \\
\end{array}$} \\
\hline & $\mathrm{F}_{1}$ vs. B73 & F $_{1}$ vs. Mo17 & Overlap & Total (\%) & & & \\
\hline Ribosome ** & 148 & 129 & 89 & $188(4.68 \%)$ & $2.47 \times 10^{-5}$ & $1.58 \times 10^{-3}$ & ko03010 \\
\hline Arginine and Proline Metabolism ** & 37 & 34 & 25 & $46(1.11 \%)$ & $2.59 \times 10^{-5}$ & $1.58 \times 10^{-3}$ & ko00330 \\
\hline Spliceosome $*$ & 145 & 136 & 87 & $194(4.82 \%)$ & $4.01 \times 10^{-4}$ & $1.63 \times 10^{-2}$ & ko03040 \\
\hline
\end{tabular}


Table 5. Cont.

\begin{tabular}{|c|c|c|c|c|c|c|c|}
\hline \multirow{2}{*}{ Pathway $^{\text {a }}$} & \multicolumn{4}{|c|}{ Different Genes with Pathway Annotation (4312) } & \multirow{2}{*}{$p$-Value ${ }^{b}$} & \multirow{2}{*}{$q$-Value ${ }^{\mathrm{c}}$} & \multirow{2}{*}{$\begin{array}{c}\text { Pathway } \\
\text { ID }\end{array}$} \\
\hline & $\mathrm{F}_{1}$ vs. B73 & $\mathrm{F}_{1}$ vs. Mo17 & Overlap & Total $(\%)$ & & & \\
\hline Pyruvate Metabolism * & 32 & 41 & 25 & $49(1.69 \%)$ & $6.28 \times 10^{-4}$ & $3.80 \times 10^{-2}$ & ko00620 \\
\hline Proteasome & 33 & 25 & 19 & $39(0.90 \%)$ & $2.00 \times 10^{-3}$ & $6.09 \times 10^{-2}$ & ko03050 \\
\hline Pentose Phosphate Pathway & 16 & 24 & 13 & $27(0.63 \%)$ & $1.66 \times 10^{-3}$ & $6.70 \times 10^{-2}$ & ko00030 \\
\hline Purine Metabolism & 64 & 72 & 39 & $97(2.25 \%)$ & $9.34 \times 10^{-4}$ & $7.50 \times 10^{-2}$ & ko00230 \\
\hline RNA Degradation & 43 & 49 & 31 & $61(1.41 \%)$ & $4.38 \times 10^{-3}$ & $8.84 \times 10^{-2}$ & ko03018 \\
\hline $\begin{array}{l}\text { Porphyrin and Chlorophyll } \\
\text { Metabolism }\end{array}$ & 21 & 16 & 11 & $26(0.60 \%)$ & $3.82 \times 10^{-3}$ & $9.32 \times 10^{-2}$ & ko00860 \\
\hline Glycolysis/Gluconeogenesis & 49 & 57 & 31 & $75(1.74 \%)$ & $8.38 \times 10^{-3}$ & $1.04 \times 10^{-1}$ & ko00010 \\
\hline
\end{tabular}

a Pathwayanalysisbased on KOBAS 2.0 (Mao et al. 2005; Wu et al. 2006; Xie et al. 2011) [46,48,49];

${ }^{\mathrm{b}} p$-value in hypergeometric test; ${ }^{\mathrm{C}}$ The $q$-value is similar to the well known $p$-value, except it is a measure of significance in terms of the false discovery rate rather than the false positive rate (Storey et al. 2003) [47]; the top 10 pathways with $q$-value are listed; * pathway with $q$-value $<0.05$ is considered as significant; ** pathway with $q$-value $<0.01$ is considered as extreme significant.

In the four significant pathways, 188 different genes $(4.68 \%)$ were detected in the ribosome pathway, 46 different genes (1.11\%) in the arginine and proline metabolism pathway, $194(4.82 \%)$ in the spliceosome pathway and $49(1.69 \%)$ in the pyruvate metabolism pathway. Approximately $50 \%$ of different genes in every pathway were differentially expressed between inbred and hybrid (Table 5). In the two significant metabolic pathways, almost all (202 out of 204) different genes showed non-additive expression patterns (HPD, UDO, LPD, or UDO). Seventeen of the 46 different genes in the arginine and proline metabolism pathway were up-regulated (with expression pattern of HPD or ODO) in the $F_{1}$ hybrid, and 26 of 46 were down-regulated (with expression pattern of LPD or UDO). As compared with parental lines, the transcriptional level of one different gene in the $F_{1}$ hybrid (GRMZM2G062142) encoding ornithine carbamoyltransferase (OTC) was up-regulated by 7- and 11.6-fold, respectively. The gene (GRMZM2G169458) encoding fatty aldehyde dehydrogenase 1 and another gene (GRMZM2G366392) encoding $S$-adenosylmethionine decarboxylase proenzyme (SAMDC) showed 4.7-/3.4-fold and 2.1-/2.9-fold higher expression in $\mathrm{F}_{1}$ hybrid than the parental lines, respectively. Simultaneously, the expression levels of another different gene in the $F_{1}$ hybrid (GRMZM2G035042) encoding IMP dehydrogenase/GMP reductase were down-regulated by 8.5- and 9.3-fold, respectively, comparing with parental lines, another gene (GRMZM2G010406) encoding argininosuccinate synthase showed significant down-regulation expression in $\mathrm{F}_{1}$ hybrid. Moreover, 50 different genes involved in the pyruvate metabolism included genes encoding glyoxylatereductase (GRMZM2G166899), phosphoenolpyruvate carboxylase1 (PEPCase 1, GRMZM2G083841), oxidoreductase (GRMZM2G118770), and Pyruvate, orthophosphate dikinase 1 (GRMZM2G097457). (Table S8).

\subsection{Resolving Transcription Factors (TFs) among Differentially Expressed Genes}

A primary objective was to identify genes that encode TFs and to determine their modes of gene action. To test this, we retrieved putative orthologs of maize genes in our differently expressed data based on information from the Ensembl Compara gene trees [50] at maizesequence.org and 
gramene.org [51]. We then queried known Arabidopsis TFs in the database of Arabidopsis Transcription Factors (http://datf.cbi.pku.edu.cn/) and identified 435 maize different genes with sequence similarities to Arabidopsis TFs between parental lines and their $\mathrm{F}_{1}$ hybrid libraries (Table $\mathrm{S} 12)$. We further interrogated these different genes using gene ontologies, InterPro domains, and known maize annotations. Of the 435 putative TFs, 11 exhibited additive gene action, and the majority of these TFs $(n=424)$ detected in this study exhibited nonadditive modes of gene expression. Most of these genes exhibited low-parent dominance $(n=138)$, high-parent dominance $(n=112)$, and underdominance $(n=112)$. However, overdominance $(n=42)$ and other gene action $(n=19)$ were also observed.

We also surveyed the differential expression of TFs across a wide range of transcript abundance in hybrid here and a mutant in RAMOSA3 (RA3) gene reported in a previous study [52], the latter of which showed an increased branching phenotype resulting from a loss of determinacy of basal spikelet pair meristems. A total of 39 differentially expressed putative TFs in our dataset were identified, and exhibited all nonadditive modes of gene expression (Table 6). Moreover, these TFs were also differentially expressed over a wide range of abundances in ra3 mutants [52]. These TFs includes several kinds of members of $\mathrm{TF}$ families associated with functions in development and meristem maintenance or identity (NAC, YABBY, GRAS and TCP), while others have roles in hormone-mediated or stress-mediated signaling by auxin (AUX/IAA), brassinosteroids (BES), or ethylene and stress (AP2/ERF). Among the 42 differentially expressed TFs, eight were characterized as AP2/ERF family proteins (Table 6). Therefore, these TFs possibly not only contribute to heterosis, but also provide insight into genetic control of branching.

Table 6. Differentially expressed maize genes were identified as putative TFs.

\begin{tabular}{|c|c|c|c|c|c|}
\hline Maize Gene ID & Annotation ${ }^{a}$ & TF Family & Significant Pattern ${ }^{b}$ & $\begin{array}{c}\text { Expression } \\
\text { Model }^{\mathrm{c}}\end{array}$ & $\begin{array}{c}\text { Regulated in } r a 3 \\
\text { Mutants }^{d}\end{array}$ \\
\hline GRMZM2G106673 & B3-domain TF & B3 & $\mathrm{Mo} 17<\mathrm{F}_{1} \approx \mathrm{B} 73$ & HPD & $\mathrm{Up}$ \\
\hline GRMZM2G177046 & Ocs element-binding factor 1 & bZIP & $\operatorname{Mo} 17<\mathrm{F}_{1} \approx \mathrm{B} 73$ & HPD & Up \\
\hline GRMZM2G102514 & BES1/BZR1 protein & BES & $\mathrm{Mo} 17<\mathrm{F}_{1} \approx \mathrm{B} 73$ & HPD & Down \\
\hline GRMZM2G172657 & Uncharacterized & GRAS & $\mathrm{Mo} 17<\mathrm{F}_{1} \approx \mathrm{B} 73$ & HPD & Down \\
\hline GRMZM2G115357 & IAA24 & AUX/IAA & $\mathrm{Mo} 17<\mathrm{F}_{1} \approx \mathrm{B} 73$ & HPD & Up \\
\hline GRMZM2G181376 & Uncharacterized & - & $\mathrm{B} 73<\mathrm{F}_{1} \approx \mathrm{Mo} 17$ & HPD & Down \\
\hline GRMZM2G173534 & Inducer of CBF expression 2 & - & $\mathrm{B} 73<\mathrm{F}_{1} \approx \mathrm{Mo} 17$ & HPD & Up \\
\hline GRMZM2G173124 & Zinc finger & Znf-C3H1 & $\mathrm{B} 73 \approx \mathrm{F}_{1}<\mathrm{Mo} 17$ & LPD & Up \\
\hline GRMZM2G138886 & Cyclin B2 & Cyclin & $\mathrm{B} 73 \approx \mathrm{F}_{1}<\mathrm{Mo} 17$ & LPD & Up \\
\hline GRMZM2G020054 & Uncharacterized & AP2/ERF & $\mathrm{Mo} 17 \approx \mathrm{F}_{1}<\mathrm{B} 73$ & LPD & $\mathrm{Up}$ \\
\hline GRMZM2G055243 & KNOX class 2 protein & KNOX & $\mathrm{Mo} 17 \approx \mathrm{F}_{1}<\mathrm{B} 73$ & LPD & $\mathrm{Up}$ \\
\hline GRMZM2G089995 & Ethylene responsive & $\mathrm{AP} 2 / \mathrm{ERF}$ & $\mathrm{Mo17} \approx \mathrm{F}_{1}<\mathrm{B} 73$ & LPD & $\mathrm{Up}$ \\
\hline GRMZM2G079825 & Pathogenesis-related & AP2/ERF & $\mathrm{Mo17} \approx \mathrm{F}_{1}<\mathrm{B} 73$ & LPD & NS \\
\hline GRMZM2G310368 & Ethylene responsive & $\mathrm{AP} 2 / \mathrm{ERF}$ & $\mathrm{Mo} 17 \approx \mathrm{F}_{1}<\mathrm{B} 73$ & LPD & Up \\
\hline GRMZM2G132185 & Pathogenesis-related & $\mathrm{AP} 2 / \mathrm{ERF}$ & $\mathrm{Mo} 17 \approx \mathrm{F}_{1}<\mathrm{B} 73$ & LPD & NS \\
\hline GRMZM2G149940 & B3 DNA binding domain & B3 & $\mathrm{Mo} 17 \approx \mathrm{F}_{1}<\mathrm{B} 73$ & LPD & Up \\
\hline GRMZM2G003927 & Ramosa1 & Znf-C2H2 & $\mathrm{B} 73 \approx \mathrm{F}_{1}<\mathrm{Mo} 17$ & LPD & Up \\
\hline GRMZM2G447406 & Progesterone receptor & - & $\mathrm{B} 73 \approx \mathrm{F}_{1}<\mathrm{Mo} 17$ & LPD & Down \\
\hline GRMZM2G102218 & YABBY protein & YABBY & $\mathrm{Mo} 17 \approx \mathrm{F}_{1}<\mathrm{B} 73$ & LPD & Up \\
\hline
\end{tabular}


Table 6. Cont.

\begin{tabular}{|c|c|c|c|c|c|}
\hline Maize Gene ID & Annotation $^{a}$ & TF Family & Significant Pattern ${ }^{\mathrm{b}}$ & $\begin{array}{l}\text { Expression } \\
\text { Model }^{\mathrm{c}}\end{array}$ & $\begin{array}{l}\text { Regulated in } r a 3 \\
\text { Mutants }{ }^{\text {d }}\end{array}$ \\
\hline GRMZM2G136769 & Ubiquitin-associated & Ubiquitin & $\mathrm{Mo17} \approx \mathrm{F}_{1}<\mathrm{B} 73$ & LPD & Up \\
\hline GRMZM2G165972 & Heat shock factor (HSF)-type & HSF & $\mathrm{Mo} 17 \approx \mathrm{F}_{1}<\mathrm{B} 73$ & LPD & Down \\
\hline GRMZM2G140474 & Tyrosine protein kinase & - & $\mathrm{Mo} 17 \approx \mathrm{F}_{1}<\mathrm{B} 73$ & LPD & $\mathrm{Up}$ \\
\hline GRMZM2G422205 & Zinc finger & Znf-C3H1 & $\mathrm{Mo} 17 \approx \mathrm{F}_{1}<\mathrm{B} 73$ & LPD & \\
\hline GRMZM2G171468 & Uncharacterized & MYB & $\mathrm{Mo} 17 \approx \mathrm{F}_{1}<\mathrm{B} 73$ & LPD & Down \\
\hline AC206951.3_FG017 & $\begin{array}{l}\text { Ethylene-responsive element } \\
\text { binding protein } 2\end{array}$ & ERF & $\mathrm{Mo17}<\mathrm{B} 73<\mathrm{F}_{1}$ & ODO & Up \\
\hline GRMZM2G081012 & $\begin{array}{l}\text { Transcription initiation } \\
\text { factor IID, } 18 \mathrm{kD} \text { subunit } \\
\text { family protein }\end{array}$ & TFIID-18 & $\mathrm{B} 73 \approx \mathrm{Mo} 17<\mathrm{F}_{1}$ & ODO & Up \\
\hline GRMZM2G014653 & NAC protein 48 & NAC & $\mathrm{B} 73 \approx \mathrm{Mo} 17<\mathrm{F}_{1}$ & ODO & Up \\
\hline GRMZM2G127379 & NAM containing & NAC & $\mathrm{B} 73 \approx \mathrm{Mo} 17<\mathrm{F}_{1}$ & ODO & Up \\
\hline GRMZM2G061487 & DRE binding factor 1 & $\mathrm{AP} 2 / \mathrm{ERF}$ & $\mathrm{F}_{1}<\mathrm{Mo} 17<\mathrm{B} 73$ & UDO & Up \\
\hline GRMZM2G431157 & Zinc finger, $\mathrm{C}_{2} \mathrm{H}_{2}$-type & $\begin{array}{l}\text { Znf_C2H2- } \\
\text { like }\end{array}$ & $\mathrm{F}_{1}<\mathrm{B} 73 \approx \mathrm{Mo} 17$ & UDO & Up \\
\hline GRMZM2G307119 & Branched silkless1 & AP2/ERF & $\mathrm{F}_{1}<\mathrm{B} 73 \approx \mathrm{Mo} 17$ & UDO & Up \\
\hline GRMZM2G144275 & bHLH transcription factor & HLH & $\mathrm{F}_{1}<\mathrm{B} 73 \approx \mathrm{Mo17}$ & UDO & Up \\
\hline GRMZM2G132367 & HDZipI-1 & HD-Zip & $\mathrm{F}_{1}<\mathrm{B} 73 \approx \mathrm{Mo} 17$ & UDO & $\mathrm{Up}$ \\
\hline GRMZM2G105266 & Pathogenesis-related & AP2/ERF & $\mathrm{F}_{1}<\mathrm{B} 73 \approx \mathrm{Mo} 17$ & UDO & NS \\
\hline GRMZM2G381395 & $\begin{array}{l}\text { DNA-directed } \\
\text { RNA polymerase }\end{array}$ & - & $\mathrm{F}_{1}<\mathrm{B} 73 \approx \mathrm{Mo} 17$ & UDO & Down \\
\hline GRMZM2G453424 & $\begin{array}{c}\text { Uncharacterized } \\
\text { DNA-directed RNA }\end{array}$ & HRDC_like & $\mathrm{F}_{1}<\mathrm{B} 73 \approx \mathrm{Mo} 17$ & UDO & Up \\
\hline GRMZM2G118113 & $\begin{array}{c}\text { polymerase II } \\
8.2 \mathrm{kDa} \text { polypeptide }\end{array}$ & $\mathrm{EF}$ & $\mathrm{F}_{1}<\mathrm{B} 73 \approx \mathrm{Mo} 17$ & UDO & Up \\
\hline GRMZM2G017606 & SHI & SHI & $\mathrm{F}_{1}<\mathrm{B} 73 \approx \mathrm{Mo} 17$ & UDO & Up \\
\hline GRMZM2G039889 & Cold acclimation protein & - & $\mathrm{F}_{1}<\mathrm{B} 73 \approx \mathrm{Mo} 17$ & UDO & $\mathrm{Up}$ \\
\hline GRMZM2G088309 & Drooping leaf & YABBY & $\mathrm{F}_{1}<\mathrm{B} 73 \approx \mathrm{Mo} 17$ & UDO & Up \\
\hline GRMZM2G078077 & TCP domain protein & $\mathrm{TCP}$ & Mo $17<\mathrm{F}_{1}<\mathrm{B} 73$ & Other & Up \\
\hline GRMZM2G404426 & Zinc finger & Znf-PHD & $\mathrm{B} 73<\mathrm{F}_{1}<\mathrm{Mo17}$ & Other & Up \\
\hline
\end{tabular}

${ }^{a}$ Annotations are based on Ensembl gene descriptions at maizesequence.org, gene build5b.60; ${ }^{\mathrm{b}}$ FDR $\leq 0.001$ and the absolute value of $\log _{2}$ Ratio $\geq 1$; ${ }^{\mathrm{c}}$ Gene expression of $\mathrm{F}_{1}$ hybrid is classified into multiple patterns. HPD indicates high-parent dominance; LPD, low-parent dominance; ODO, over-dominance; UDO, under-dominance; ND, undistinguishable model from additivity and non-additivity; ${ }^{d}$ reference Eveland et al. (2010) [52]. NS, Not significant.

\subsection{A Significant Number of Genes Were Expressed in Only One Inbred Line or Absent in both}

\section{Inbred Lines}

A total of $5660(24.8 \%)$ genes with no detectable expression in one inbred line or both two inbred lines were identified (Table 7). In other word, there are a significant number of genes that displayed presence-absence expression patterns. Among these genes, 46.4\% (2624 of 5660) genes exhibited an expression pattern that was present in B73, and absent in Mo17, while some other $34.8 \%$ (1971 out of 
5660) genes exhibited a similar expression pattern that present in Mo17, and absent in B73 (Tables 7 and S13). Moreover, these genes expressed in only one inbred line also displayed presence-absence expression patterns in their hybrid. Specifically, regarding the genes present in B73 and absent in Mo17, the ratio of genes present to genes absent in their hybrid was approximately $0.56: 1$, while the ratio of genes present to genes absent in their hybrid was approximately $1.31: 1$ for other genes present in Mo17 and absent in B73. In combination, these results suggested that the majority of genes that were expressed in only one inbred line exhibited parental effects on gene expression levels, and presence-absence expression patterns of some genes may be related with presence/absence variations in maize genes [53] (See the following analysis). Surprisingly, it was found that $18.8 \%$ (1065 of 5660) genes were not expressed in the two inbred parents. However, these genes were expressed in their hybrid (Tables 7 and S13). Additionally, nine genes expressed in only one inbred line were also found in previous study by Stupar et al. [14], and these results in two studies were consistent. For instance, GRMZM2G037255 (corresponding accession \#CF629797) and GRMZM2G152258 (accession \#BM073080) absent in both inbred lines in our study were not detected in both B73 and Mo17 by PCR in the study of Stupar et al. [14] (Table S13). However, the other 106 genes were expressed in only one inbred in Stupar's study [14], and were expressed in both B73 and Mo17 in present study.

Table 7. Genes with no detectable expression in one inbred line or both two inbred lines.

\begin{tabular}{ccccccc}
\hline Class & $\begin{array}{c}\text { No. of Genes } \\
\text { Present in B73, } \\
\text { Absent in Mo17 }\end{array}$ & $\begin{array}{c}\text { \% of } \\
\text { Genes }\end{array}$ & $\begin{array}{c}\text { No. of Genes } \\
\text { Present in Mo17, } \\
\text { Absent in B73 }\end{array}$ & $\begin{array}{c}\text { \% of } \\
\text { Genes }\end{array}$ & $\begin{array}{c}\text { No. of Genes } \\
\text { Absent in both } \\
\text { B73 and Mo17 }\end{array}$ & $\begin{array}{c}\text { \% of } \\
\text { Genes }\end{array}$ \\
\hline $\begin{array}{c}\text { No. of genes } \\
\text { present in their hybrid } \\
\text { No. of genes }\end{array}$ & 942 & 16.6 & 1117 & 19.7 & 1065 & 18.8 \\
$\begin{array}{c}\text { absent in their hybrid } \\
\text { Total }\end{array}$ & 1,682 & 29.7 & 854 & 15.1 & 0 & 0 \\
\hline
\end{tabular}

\subsection{Analysis of Presence/Absence Variation (PAVs) Genes Action in Maize Hybrids}

Presence/absence variations (PAVs) have been described in maize genes [53], and most of the PAVs reflected true differences in gene content between the B73 and Mo17 genomes in recent research. Because of the availability of a complete list of PAV genes identified by Lai et al. [54], the B73 $\times$ Mo17 cross was first examined regarding PAV genes between the two parental lines and its relation to different gene expression among the three genotypes. As shown in Table S14, there were 104 PAV genes between the two parental lines, and only 37 PAV genes (35.6\%) were mapped by identified tags in three libraries. Interestingly, most of these mapped genes products were hypothetical proteins, and the other 55.8\% (58 of 104) of genes were unknown proteins except for nine genes including terpene synthase and ZCN20 (Table S14). Of these mapped genes, 45.9\% (17 of 37) genes were expressed in only B73. Six genes expressed in only one inbred line were also not expressed in their hybrid. What was more puzzling is there were three genes which were not expressed in the two inbred parents, nevertheless, expressed in their hybrid. Interestingly, among the 37 PAV genes, the expression levels (TPM) of $30 \%$ genes in $\mathrm{F}_{1}$ hybrid was the same as that of B73 or Mo17, and $50 \%$ showed higher or lower expression in $\mathrm{F}_{1}$ hybrid than both the parental lines (Table S15). 
However, only five PAV genes were identified with significantly differential expression with HPD or LPD (Table S14).

\section{Discussion}

In this study, we assayed genome-wide patterns of gene expression of the maize ear at an early flower differentiation stage among two maize elite inbred lines (B73 and Mo17) and their $\mathrm{F}_{1}$ hybrid $($ B73 $\times$ Mo17) using Solexa's digital gene expression (DGE) system, a tag-based novel high-throughput transcriptome deep sequencing method. Given the nature of the DGE system, we have pooled biological replicates from three varieties for each group to make representative samples for deep sequencing analysis. We obtained a sequencing depth of approximately 4.2 million tags per library (Table 1) and found 22,789 genes expressed collectively except for putative new transcripts found in the study. Levels of some genes not expressed in the present study were responsive to abiotic stress, e.g., aquaporin PIP1-6 gene (GRMZM2G136032), MADS-box transcription factor 14 gene (GRMZM2G137510), beta-fructofuranosidase 1 precursor gene (GRMZM2G394450), etc., were induced by heavy metal $\mathrm{Pb}$ treatment in one recent study [55]. Interestingly, we found evidence for bidirectional transcription in all datasets. By comparison of all libraries, the ratio of sense to antisense strands of the transcripts was approximately 1.3:1, which suggested that not only a high number of antisense expressions, but also the transcriptional regulation in the young ear development acted most strongly on the sense strand. A similar observation was also reported in a recent study [56]. Furthermore, approximately $20 \%$ of genes with no detectable expression in one inbred line were identified. These genes may exhibit parental effects on gene expression levels.

Based on our digital gene expression analysis, approximately $37.8 \%$ of genes exhibited differential expression between every two genotypes in the B73 $\times$ Mo17 hybrid cross. QPCR validation was performed both on the same pooled material that was used for deep sequencing and on independent RNA extractions from each sample, and almost all confirmed the direction of change detected by DGE analysis (Figure 3). These different genes exhibited additive and non-additive expression patterns (Figure 2). A small fraction of DGs (8.9\%, 767 of 8621) exhibited a mode of gene action that was indistinguishable from additivity, which is similar to recent studies in maize [14,23,25,31]. Several studies reported more nonadditively expressed genes, including many with $\mathrm{F}_{1}$ expression levels outside the parental range [11-13,23,27]. Among those nonadditively expressed genes, the proportion of genes with clear over and under-dominant gene action were $12.6 \%$ and $22.8 \%$, respectively, which is similar to results from prior studies [12,15,16,31,57]. Additionally, we further compared the differences of modes of gene action between Swanson-Wagner's study [31] and the present study. A total of 8621 different gene BLAST searches were performed using 1367 ESTs as queries. Four hundred and twenty-one different genes were matched by 547 ESTs with high-scoring segment pairs (Table S15), but only a small number of these genes (75/421) had the same mode of gene action between the two studies. Thus, different global expression patterns in different tissues or developmental stages might prevail. Our results support that multiple molecular mechanisms (dominance and overdominance modes) contribute to heterosis, which is consistent with previous reports [21,31].

No consensus has yet been reached about the genetic basis of heterosis [58]. However, some mechanisms were supported by the observations that sequence polymorphism in promoter alleles 
between inbred lines preferentially occurred in those differentially transcribed genes [16]. When two alleles are exposed to a common trans-acting factor, cis-elements in hybrids might differentially interact with gene regulators, resulting in allele-specific gene expression [14,16,59]. This undoubtedly is one of the causes of gene-expression changes in hybrids. There was evidence that phenotypes in hybrids resulted from the dosage effect of such regulatory genes [60]. In this study, we found 424 putative TFs, exhibiting differential expression in the hybrid compared with either parent, in agreement with recent studies [16]. Remarkably, about $9.2 \%$ of these TFs were also differentially expressed in ra3 mutants (Table 6) and many of the differentially expressed genes that could be mapped onto metabolic pathways were associated with primary carbohydrate biosynthesis and degradation, respiration, and energy production as well as redox and nitrogen cycling processes [52]. In conclusion, the expression of TFs in maize hybrids might be important for allele-specific gene expression in heterosis. Another important finding in a recent study is that many SNPs, indel polymorphisms (IDPs) and PAV genes identified between the B73 and Mo17 genome [54] were consistent with the occurrence of insertion/deletion (indel) variants in 5' regions between the alleles of genes that are differentially expressed in different rice strains [16]. We also analyzed the expression of all 104 PAV genes between the two parental lines by DGE data. Of these genes, 67 PAV genes (64.4\%) did not express in both B73 and Mo17, possibly because these genes were nonfunctional. For another 37 PAV genes, most (33/37) of their gene products were hypothetical proteins, unknown proteins, or no significant BLAST hits were obtained by using an $e$-value cutoff of $1 \times 10^{-5}$, and almost $50 \%$ of these genes were expressed in both inbred lines and their hybrid. Specially, eight PAV genes were expressed only in one inbred line and their hybrid, which possibly contributed to heterosis, because the phenomenon conformed to the previous assumption that inbred lines with large differences in gene content could complement one another [54]. Moreover, the expression levels of approximately $30 \%$ ( 9 of 37) of these genes in the $\mathrm{F}_{1}$ hybrid was the same as that of B73 or Mo17, and $50 \%$ showed higher or lower expression in $F_{1}$ hybrid than both the parental lines. Interestingly, only five PAV genes were identified with significantly differential expression in the hybrid. It suggests that the expression of only a part of PAV genes was consistent with the complementation hypothesis. In conclusion, it is unlikely that heterosis is the result of any single mechanism [58,61].

\section{Experimental Section}

\subsection{Plant Growth and RNA Isolation}

The hybrid corn combination, $\mathrm{B} 73 \times \mathrm{Mo17}\left(\mathrm{F}_{1}\right.$ and its parental lines, B73 and Mo17), was originally obtained from Thomas Lübberstedt (Iowa State University, Ames, IA, USA). The materials were offered for high-throughput sequencing and quantitative real time PCR (qRT-PCR) analysis. The inbred lines were cultivated at the experimental station of Sichuan Agricultural University, Chengdu, for seed propagation of the inbreds, and for production of B73 $\times$ Mo17 hybrid seed. Kernels of the combination were grown in environmentally controlled growth chambers that provided $15 \mathrm{~h}$ of light $\left(25^{\circ} \mathrm{C}\right)$ and $9 \mathrm{~h}$ of dark $\left(20^{\circ} \mathrm{C}\right)$ as described previously. Light intensity was $\approx 650-800 \mu \mathrm{mol} \cdot \mathrm{m}^{-2} \cdot \mathrm{s}^{-1}$. Ears of 10 random healthy plants at early flower differentiation stages were manually collected as a pool for each genotype as described previously [62]. In brief, ears at stage 3 (three stamen primordia 
and one pistil primordium can be observed) were collected according to the plant features (number of visible leaves, leaf age index, number of unfolded and folded leaves) combined with microscopic observation. At stage 3, the number of visible leaves, leaf age index, and the number of unfolded and folded leaves are 19,65\%, 13.7 and 5, respectively. Morphological observations of ears at stage 3 in two inbred lines and their $F_{1}$ were almost the same as that in another inbred line X178 reported previously [62]. After separately grinding meristems in liquid nitrogen, RNA were extracted for constructing three digital gene expression libraries, and quantitative real-time PCR validation from $\approx 5 \mathrm{~g}$ of frozen tissue by using TRIzol reagent (Invitrogen, Carlsbad, CA, USA) according to the manufacturer's instructions.

\subsection{Digital Gene Expression Library Preparation and Sequencing}

Tag library preparation for the three genotypes (B73, Mo17 and their $\mathrm{F}_{1}$ ) was performed in parallel by using the Illumina gene expression sample preparation kit as described previously [63]. An extract of $6 \mu \mathrm{g}$ of total RNA was obtained and treated with Oligo (dT) magnetic bead adsorption to purify mRNA. Oligo (dT) was then used as a primer to synthesize the first- and second-strand cDNA. The $5^{\prime}$ ends of tags were generated by two endonucleases NlaIII or DpnII. The bead-bound cDNA was subsequently digested with restriction enzyme NlaIII, which recognizes and cuts off the CATG sites. The fragments apart from the 3' cDNA fragments connected to Oligo (dT) beads were washed away and the Illumina adaptor 1 was ligated to the sticky $5^{\prime}$ end of the digested bead-bound cDNA fragments. The junction of Illumina adaptor 1 and CATG site is the recognition site of MmeI, which is a type of Endonuclease with separate recognition and digestion sites. It cuts $17 \mathrm{bp}$ downstream of the CATG site, producing tags with adaptor 1. After removing 3' fragments with magnetic beads by precipitation, Illumina adaptor 2 was ligated to the $3^{\prime}$ ends of tags, acquiring tags with different adaptors at both ends to form a tag library. After 15 cycles of linear PCR amplification, $95 \mathrm{bp}$ fragments were purified by $6 \%$ TBE PAGE Gel electrophoresis. After denaturation, the single-chain molecules were fixed onto the Illumina Sequencing flowcell. Each molecule grows into a single-molecule cluster sequencing template through in situ amplification. The four types of nucleotides were labeled by four colors, and added to perform sequencing by synthesis (SBS) [64]. Each tunnel will generate millions of raw reads with sequencing length of $35 \mathrm{bp}$.

\subsection{Quantitative RT-PCR and Gene Expression Analysis}

In order to verify a sample of genes that exhibited statistically significant differential expression in the analysis of DGE data, we used quantitative real time PCR analysis. The RNA samples used for the qRT-PCR assays were the same as for the DGE experiments from 10 biological replicates. First, $1 \mu \mathrm{g}$ of RNA was treated with RNase-free DNase (Promega, Madison, WI, USA), and cDNA was synthesized with PrimeScript RT reagent kit (TaKaRa, Tokyo, Japan). Then, qRT-PCR of 30 differentially expressed genes (Table S16), which were involved in arginine and proline metabolism, pyruvate metabolism or other genes, were performed using the SYBR PremixExTaq ${ }^{\mathrm{TM}}$ protocol (TaKaRa, Tokyo, Japan) on an Applied Biosystems 7500 Real-Time PCR System (Applied Biosystems, Foster City, CA, USA). For each sample, measurements were performed in triplicate, and 
the average cycle thresholds $\left(C_{\mathrm{t}}\right)$ were used to determine fold-change. $18 \mathrm{~S}$ rRNA was employed as an endogenous control. The results were calculated using the $2^{-\Delta \Delta C t}$ method [65].

\subsection{Analysis and Mapping of Digital Gene Expression Tags}

Raw sequencing image data were transformed by base calling into sequences. These raw data reads were stored in FASTQ format, and their analysis conducted as described by Qin et al. [63]. In brief, prior to mapping to the reference database, all sequences were filtered to trim the 3 ' adaptor sequence, filter empty tags (reads with only 3' adaptor sequences but no tags) and low-quality tags containing Ns, and remove tags which are too long or too short. A virtual library containing all possible CATG +17 base-length sequences of the maize genome database (AGPv2, release 5b.60) [43] was utilized. All clean tags were mapped to the reference sequences and a mismatch of only $1 \mathrm{bp}$ was considered. Clean tags that were mapped to the maize genome reference sequences from multiple genes were filtered. The remaining clean tags were designed as unambiguous clean tags. The expression level of each gene was estimated by the frequency of clean tags and then normalized to TPM (number of transcripts per million clean tags) [66], which is a standard method and extensively used in DGE analysis [67]. KOG functional classification, Gene Ontology (GO), pathway annotation and enrichment analyses were based on the NCBI COG (http://www.ncbi.nlm.nih.gov/COG) [68], Gene Ontology Database (http://www.geneontology.org/) [69] and KEGG pathway (http://www.genome.jp/kegg/) [70], respectively. When we investigate pathways in which DGs were involved and enriched, $q$-value was used for aided identification according to the previous description [47].

\subsection{Identification of Differentially Expressed Genes}

To examine differential expression across samples (B73, Mo17 and their hybrid), the number of raw clean tags in each library was normalized to TPM to obtain normalized gene expression levels. Detection of different tags across samples were performed as previously described [71]. The false discovery rate $(5 \%)$ is controlled by the Benjamini and Hochberg's procedure [72]. After multiple testing between pairwise comparisons, we use "FDR $\leq 0.001$ and the absolute value of $\log _{2}$ Ratio $\geq 1$ " as the threshold to judge the significance of gene expression difference. More stringent criteria with smaller FDR and bigger fold-change values were used to identify different genes.

In the present study, the same strategy was performed in a linear-in-genotype contrast when $F_{1}$ genotype was compared to the two parental lines as described by Zhang et al. [16]. The genes with "FDR $\leq 0.001$ and the absolute value of $\log _{2}$ Ratio $\geq 1$ " were regarded as non-additivity, when the genes with "FDR $>0.001$ and the absolute value of $\log _{2}$ Ratio $<1$ " were regarded as not statistically significantly different from additivity. To classify the genes further, the high-parent dominant genes and low-parent dominant genes were identified from the non-additive group based on the criterion, that the $F_{1}$ genotype was significantly different from one parent and not significantly different from another parent. From the non-additive group, the expression of genes was identified as over- or under-dominant, when expression in the $F_{1}$ genotype was significantly higher or lower than in both inbred parents, respectively. 


\section{Conclusions}

Our analysis revealed 17,128 genes expressed in these three genotypes and 22,789 genes expressed collectively in the present study. Approximately $38 \%$ of the genes were differentially expressed in early maize ear inflorescences from heterotic cross, including many transcription factor genes and some presence/absence variations (PAVs) genes, and exhibited multiple modes of gene action. Additionally, a significant number of genes were expressed in only one inbred line or absent in both inbred lines. Comparison of the differences of modes of gene action between previous studies and the present study revealed only small number of different genes had the same modes of gene action in both maize seedlings and ear inflorescences, it might of be an indication that, in different tissues or developmental stages, different global expression patterns might prevail, which might nevertheless be related to heterosis. Our results support the hypothesis that multiple molecular mechanisms (dominance and overdominance modes) contribute to heterosis.

\section{Acknowledgments}

This work was supported by National modern agricultural industry technology system, the Major Project of China on New varieties of GMO Cultivation (2011ZX08003-003), National Natural Science Foundation of China (30201639), and the national Hi-Tec program of China (2012AA10307).

\section{Author Contributions}

H.D., G.P., C.Q. and Z.Z. designed the study; C.Q., X.L., L.L., J.G., H.L., Y.S. and M.Z. performed the analyses; C.Q and Z.C. conducted qRT-PCR; H.D., C.Q. and T.L. drafted the manuscript. All of the authors critically revised and provided final approval of this manuscript.

\section{Conflicts of Interest}

The authors declare no conflict of interest.

\section{References}

1. Shull, G.H. The composition of a field of maize. J. Hered. 1908, 4, 296-301.

2. Bruce, A.B. The Mendelian theory of heredity and the augmentation of vigor. Science 1910, 32, 627.

3. Jones, D.F. Dominance of linked factors as a means of accounting for heterosis. Genetics 1917, 6 , 466-479.

4. Davenport, C.B. Degeneration, albinism and inbreeding. Science 1908, 28, 454.

5. East, E.M. Heterosis. Genetics 1936, 21, 375-397.

6. Birchler, J.A.; Auger, D.L.; Riddle, N.C. In search of the molecular basis of heterosis. Plant Cell 2003, 15, 2236-2239.

7. Yu, S.B.; Li, J.X.; Xu, C.G.; Tan, Y.F.; Gao, Y.J.; Li, X.H.; Zhang, Q.F.; Saghai Maroof, M.A. Importance of epistasis as the genetic basis of heterosis in an elite rice hybrid. Proc. Natl. Acad. Sci. USA 1997, 99, 9226-9231. 
8. Li, Z.K.; Luo, L.J.; Mei, H.W.; Wang, D.L.; Shu, Q.Y.; Tabien, R.; Zhong, D.B.; Ying, C.S.; Stansel, J.W.; Khush, G.S.; et al. Overdominant epistatic loci are the primary genetic basis of inbreeding depression and heterosis in rice. I. Biomass and grain yield. Genetics 2001, 158, $1737-1753$.

9. Xiao, J.; Li, J.; Yuan, L.; Tanksley, S.D. Dominance is the major genetic basis of heterosis in rice as revealed by QTL analysis using molecular markers. Genetics 1995, 140, 745-754.

10. Stuber, C.W.; Lincoln, S.E.; Wolff, D.W.; Helentjaris, T.; Lander, E.S. Identification of genetic factors contributing to heterosis in a hybrid from two elite maize inbred lines using molecular markers. Genetics 1992, 132, 823-839.

11. Auger, D.L.; Gray, A.D.; Ream, T.S.; Kato, A.; Coe, E.H., Jr.; Birchler, J.A. Nonadditive gene expression in diploid and triploid hybrids of maize. Genetics 2005, 169, 389-397.

12. Hoecker, N.; Keller, B.; Muthreich, N.; Chollet, D.; Descombes, P.; Piepho, H.P.; Hochholdinger, F. Comparison of maize (Zea mays L.) $\mathrm{F}_{1}$-hybrid and parental inbred line primary root transcriptomes suggests organ-specific patterns of nonadditive gene expression and conserved expression trends. Genetics 2008, 179, 1275-1283.

13. Song, R.; Messing, J. Gene expression of a gene family in maize based on noncollinear haplotypes. Proc. Natl. Acad. Sci. USA 2003, 100, 9055-9060.

14. Stupar, R.M.; Springer, N.M. cis-Transcriptional variation in maize inbred lines B73 and Mo17 leads to additive expression patterns in the $\mathrm{F}_{1}$ hybrid. Genetics 2006, 173, 2199-2210.

15. Vuylsteke, M.; van Eeuwijk, F.; van Hummelen, P.; Kuiper, M.; Zabeau, M. Genetic analysis of variation in gene expression in Arabidopsis thaliana. Genetics 2005, 171, 1267-1275.

16. Zhang, H.Y.; He, H.; Chen, L.B.; Li, L.; Liang, M.Z.; Wang, X.F.; Liu, X.G.; He, G.M.; Chen, R.S.; Ma, L.G.; et al. A genome-wide transcription analysis reveals a close correlation of promoter INDEL polymorphism and heterotic gene expression in rice hybrids. Mol. Plant 2008, 1, $720-731$.

17. Guo, M.; Yang, S.; Rupe, M.; Hu, B.; Bickel, D.; Arthur, L.; Smith, O. Genome-wide allele-specific expression analysis using Massively Parallel Signature Sequencing (MPSS ${ }^{\mathrm{TM}}$ ) Reveals cis- and trans-effects on gene expression in maize hybrid meristem tissue. Plant Mol. Biol. 2008, 66, 551-563.

18. Chen, X.; Li, M.; Shi, J.; Fu, D.; Qian, W.; Zou, J.; Zhang, C.; Meng, J. Gene expression profiles associated with intersubgenomic heterosis in Brassica napus. Theor. Appl. Genet. 2008, 117, 1031-1040.

19. Zhuang, Y.; Adams, K.L. Extensive allelic variation in gene expression in populus $\mathrm{F}_{1}$ hybrids. Genetics 2007, 177, 1987-1996.

20. Jahnke, S.; Sarholz, B.; Thiemann, A.; Kühr, V.; Gutiérrez-Marcos, J.; Geiger, H.; Piepho, H.-P.; Scholten, S. Heterosis in early seed development: A comparative study of $F_{1}$ embryo and endosperm tissues 6 days after fertilization. Theor. Appl. Genet. 2010, 120, 389-400.

21. Pea, G.; Ferron, S.; Gianfranceschi, L.; Krajewski, P.; Enrico Pe, M. Gene expression non-additivity in immature ears of a heterotic $\mathrm{F}_{1}$ maize hybrid. Plant Sci. 2008, 174, 17-24.

22. Riddle, N.; Jiang, H.; An, L.; Doerge, R.; Birchler, J. Gene expression analysis at the intersection of ploidy and hybridity in maize. Theor. Appl. Genet. 2010, 120, 341-353. 
23. Guo, M.; Rupe, M.A.; Yang, X.; Crasta, O.; Zinselmeier, C.; Smith, O.S.; Bowen, B. Genome-wide transcript analysis of maize hybrids: Allelic additive gene expression and yield heterosis. Theor. Appl. Genet. 2006, 113, 831-845.

24. Paschold, A.; Marcon, C.; Hoecker, N.; Hochholdinger, F. Molecular dissection of heterosis manifestation during early maize root development. Theor. Appl. Genet. 2010, 120, 383-388.

25. Meyer, S.; Pospisil, H.; Scholten, S. Heterosis associated gene expression in maize embryos 6 days after fertilization exhibits additive, dominant and overdominant pattern. Plant Mol. Biol. 2007, 63, 381-391.

26. Stupar, R.M.; Gardiner, J.M.; Oldre, A.G.; Haun, W.J.; Chandler, V.L.; Springer, N.M. Gene expression analyses in maize inbreds and hybrids with varying levels of heterosis. BMC Plant Biol. 2008, 8, 33.

27. Uzarowska, A.; Keller, B.; Piepho, H.P.; Schwarz, G.; Ingvardsen, C.; Wenzel, G.; Lubberstedt, T. Comparative expression profiling in meristems of inbred-hybrid triplets of maize based on morphological investigations of heterosis for plant height. Plant Mol. Biol. 2007, 63, 21-34.

28. Sun, Q.; Wu, L.; Ni, Z.; Meng, F.; Wang, Z.; Lin, Z. Differential gene expression patterns in leaves between hybrids and their parental inbreds are correlated with heterosis in a wheat diallel cross. Plant Sci. 2004, 166, 651-657.

29. Swanson-Wagner, R.A.; DeCook, R.; Jia, Y.; Bancroft, T.; Ji, T.; Zhao, X.; Nettleton, D.; Schnable, P.S. Paternal dominance of trans-eQTL influences gene expression patterns in maize hybrids. Science 2009, 326, 1118-1120.

30. Wei, G.; Tao, Y.; Liu, G.; Chen, C.; Luo, R.; Xia, H.; Gan, Q.; Zeng, H.; Lu, Z.; Han, Y.; et al. A transcriptomic analysis of superhybrid rice LYP9 and its parents. Proc. Natl. Acad. Sci. USA 2009, 106, 7695-7701.

31. Swanson-Wagner, R.A.; Jia, Y.; DeCook, R.; Borsuk, L.A.; Nettleton, D.; Schnable, P.S. All possible modes of gene action are observed in a global comparison of gene expression in a maize $\mathrm{F}_{1}$ hybrid and its inbred parents. Proc. Natl. Acad. Sci. USA 2006, 103, 6805-6810.

32. Song, G.S.; Zhai, H.L.; Peng, Y.G.; Zhang, L.; Wei, G.; Chen, X.Y.; Xiao, Y.G.; Wang, L.; Chen, Y.J.; Wu, B.; et al. Comparative transcriptional profiling and preliminary study on heterosis mechanism of super-hybrid rice. Mol. Plant 2010, 3, 1012-1025.

33. Chen, Z.J. Genetic and epigenetic mechanisms for gene expression and phenotypic variation in plant polyploids. Annu. Rev. Plant Biol. 2007, 58, 377-406.

34. Ha, M.; Lu, J.; Tian, L.; Ramachandran, V.; Kasschau, K.D.; Chapman, E.J.; Carrington, J.C.; Chen, X.; Wang, X.J.; Chen, Z.J. Small RNAs serve as a genetic buffer against genomic shock in Arabidopsis interspecific hybrids and allopolyploids. Proc. Natl. Acad. Sci. USA 2009, 106, 17835-17840.

35. He, G.; Zhu, X.; Elling, A.A.; Chen, L.; Wang, X.; Guo, L.; Liang, M.; He, H.; Zhang, H.; Chen, F.; et al. Global epigenetic and transcriptional trends among two rice subspecies and their reciprocal hybrids. Plant Cell 2010, 22, 17-33.

36. Liu, H.; Qin, C.; Chen, Z.; Zuo, T.; Yang, X.; Zhou, H.; Xu, M.; Cao, S.; Shen, Y.; Lin, H.; et al. Identification of miRNAs and their target genes in developing maize ears by combined small RNA and degradome sequencing. BMC Genomics 2014, 15, 25. 
37. Hollick, J.B.; Chandler, V.L. Epigenetic allelic states of a maize transcriptional regulatory locus exhibit overdominant gene action. Genetics 1998, 150, 891-897.

38. Zhao, X.; Chai, Y.; Liu, B. Epigenetic inheritance and variation of DNA methylation level and pattern in maize intra-specific hybrids. Plant Sci. 2007, 172, 930-938.

39. Ni, Z.; Kim, E.D.; Ha, M.; Lackey, E.; Liu, J.; Zhang, Y.; Sun, Q.; Chen, Z.J. Altered circadian rhythms regulate growth vigour in hybrids and allopolyploids. Nature 2009, 457, 327-331.

40. Wang, X.; Cao, H.; Zhang, D.; Li, B.; He, Y.; Li, J.; Wang, S. Relationship between differential gene expression and heterosis during ear development in maize (zea mays L.). J. Genet. Genomics 2007, 34, 160-170.

41. Li, R.; Yu, C.; Li, Y.; Lam, T.W.; Yiu, S.M.; Kristiansen, K.; Wang, J. SOAP2: An improved ultrafast tool for short read alignment. Bioinformatics 2009, 25, 1966-1967.

42. Li, P.; Ponnala, L.; Gandotra, N.; Wang, L.; Si, Y.; Tausta, S.L.; Kebrom, T.H.; Provart, N.; Patel, R.; Myers, C.R.; et al. The developmental dynamics of the maize leaf transcriptome. Nat. Genet. 2010, 42, 1060-1067.

43. Schnable, P.S.; Ware, D.; Fulton, R.S.; Stein, J.C.; Wei, F.; Pasternak, S.; Liang, C.; Zhang, J.; Fulton, L.; Graves, T.A.; et al. The B73 maize genome: Complexity, diversity, and dynamics. Science 2009, 326, 1112-1115.

44. Ashburner, M.; Ball, C.A.; Blake, J.A.; Botstein, D.; Butler, H.; Cherry, J.M.; Davis, A.P.; Dolinski, K.; Dwight, S.S.; Eppig, J.T.; et al. Gene ontology: Tool for the unification of biology—The gene ontology consortium. Nat. Genet. 2000, 25, 25-29.

45. Carbon, S.; Ireland, A.; Mungall, C.J.; Shu, S.; Marshall, B.; Lewis, S. AmiGO: Online access to ontology and annotation data. Bioinformatics 2009, 25, 288-289.

46. Xie, C.; Mao, X.; Huang, J.; Ding, Y.; Wu, J.; Dong, S.; Kong, L.; Gao, G.; Li, C.-Y.; Wei, L. KOBAS 2.0: A web server for annotation and identification of enriched pathways and diseases. Nucleic Acids Res. 2011, 39, W316-W322.

47. Storey, J.D.; Tibshirani, R. Statistical significance for genomewide studies. Proc. Natl. Acad. Sci. USA 2003, 100, 9440-9445.

48. Mao, X.; Cai, T.; Olyarchuk, J.G.; Wei, L. Automated genome annotation and pathway identification using the KEGG Orthology $(\mathrm{KO})$ as a controlled vocabulary. Bioinformatics 2005, 21, 3787-3793.

49. Wu, J.; Mao, X.; Cai, T.; Luo, J.; Wei, L. KOBAS server: A web-based platform for automated annotation and pathway identification. Nucleic Acids Res. 2006, 34, W720-W724.

50. Vilella, A.J.; Severin, J.; Ureta-Vidal, A.; Heng, L.; Durbin, R.; Birney, E. EnsemblCompara GeneTrees: Complete, duplication-aware phylogenetic trees in vertebrates. Genome Res. 2009, 19, 327-335.

51. Liang, C.; Jaiswal, P.; Hebbard, C.; Avraham, S.; Buckler, E.S.; Casstevens, T.; Hurwitz, B.; McCouch, S.; Ni, J.; Pujar, A.; et al. Gramene: A growing plant comparative genomics resource. Nucleic Acids Res. 2008, 36, D947-D953.

52. Eveland, A.L.; Satoh-Nagasawa, N.; Goldshmidt, A.; Meyer, S.; Beatty, M.; Sakai, H.; Ware, D.; Jackson, D. Digital gene expression signatures for maize development. Plant Physiol. 2010, 154, 1024-1039. 
53. Fu, H.; Dooner, H.K. Intraspecific violation of genetic colinearity and its implications in maize. Proc. Natl. Acad. Sci. USA 2002, 99, 9573-9578.

54. Lai, J.; Li, R.; Xu, X.; Jin, W.; Xu, M.; Zhao, H.; Xiang, Z.; Song, W.; Ying, K.; Zhang, M.; et al. Genome-wide patterns of genetic variation among elite maize inbred lines. Nat. Genet. 2010, 42, 1027-1030.

55. Shen, Y.; Zhang, Y.; Chen, J.; Lin, H.; Zhao, M.; Peng, H.; Liu, L.; Yuan, G.; Zhang, S.; Zhang, Z.; et al. Genome expression profile analysis reveals important transcripts in maize roots responding to the stress of heavy metal $\mathrm{Pb}$. Physiol. Plant. 2012, 147, 270-282.

56. Xiao, S.; Jia, J.; Mo, D.; Wang, Q.; Qin, L.; He, Z.; Zhao, X.; Huang, Y.; Li, A.; Yu, J.; et al. Understanding PRRSV infection in porcine lung based on genome-wide transcriptome response identified by deep sequencing. PLoS One 2010, 5, e11377.

57. Gibson, G.; Riley-Berger, R.; Harshman, L.; Kopp, A.; Vacha, S.; Nuzhdin, S.; Wayne, M. Extensive sex-specific nonadditivity of gene expression in Drosophila melanogaster. Genetics 2004, 167, 1791-1799.

58. Charlesworth, D.; Willis, J.H. The genetics of inbreeding depression. Nat. Rev. Genet. 2009, 10, 783-796.

59. Guo, M.; Rupe, M.A.; Zinselmeier, C.; Habben, J.; Bowen, B.A.; Smith, O.S. Allelic variation of gene expression in maize hybrids. Plant Cell 2004, 16, 1707-1716.

60. Birchler, J.A.; Riddle, N.C.; Auger, D.L.; Veitia, R.A. Dosage balance in gene regulation: Biological implications. Trends Genet. 2005, 21, 219-226.

61. Springer, N.M.; Stupar, R.M. Allelic variation and heterosis in maize: How do two halves make more than a whole? Genome Res. 2007, 17, 264-275.

62. Zhu, Y.; Fu, J.; Zhang, J.; Liu, T.; Jia, Z.; Wang, J.; Jin, Y.; Lian, Y.; Wang, M.; Zheng, J.; et al. Genome-wide analysis of gene expression profiles during ear development of maize. Plant Mol. Biol. 2009, 70, 63-77.

63. Qin, Y.F.; Fang, H.M.; Tian, Q.N.; Bao, Z.X.; Lu, P.; Zhao, J.M.; Mai, J.; Zhu, Z.Y.; Shu, L.L.; Zhao, L.; et al. Transcriptome profiling and digital gene expression by deep-sequencing in normal/regenerative tissues of planarian Dugesia japonica. Genomics 2011, 97, 364-371.

64. Mardis, E.R. The impact of next-generation sequencing technology on genetics. Trends Genet. 2008, 24, 133-141.

65. Livak, K.J.; Schmittgen, T.D. Analysis of relative gene expression data using real-time quantitative PCR and the $2^{-\Delta \Delta C t}$ method. Methods 2001, 25, 402-408.

66. C't Hoen, P.A.; Ariyurek, Y.; Thygesen, H.H.; Vreugdenhil, E.; Vossen, R.H.; de Menezes, R.X.; Boer, J.M.; van Ommen, G.J.B.; den Dunnen, J.T. Deep sequencing-based expression analysis shows major advances in robustness, resolution and inter-lab portability over five microarray platforms. Nucleic Acids Res. 2008, 36, e141.

67. Morrissy, A.S.; Morin, R.D.; Delaney, A.; Zeng, T.; McDonald, H.; Jones, S.; Zhao, Y.; Hirst, M.; Marra, M.A. Next-generation tag sequencing for cancer gene expression profiling. Genome Res. 2009, 19, 1825-1835.

68. Tatusov, R.L.; Fedorova, N.D.; Jackson, J.D.; Jacobs, A.R.; Kiryutin, B.; Koonin, E.V.; Krylov, D.M.; Mazumder, R.; Mekhedov, S.L.; Nikolskaya, A.N.; et al. The COG database: An updated version includes eukaryotes. BMC Bioinform. 2003, 4, 41. 
69. Consortium, T.G.O. The gene ontology project in 2008. Nucleic Acids Res. 2008, 36, D440-D444.

70. Kanehisa, M. The KEGG Database. In "In Silico" Simulation of Biological Processes; John Wiley \& Sons: Manhattan, NY, USA, 2008; pp. 91-103.

71. Marioni, J.C.; Mason, C.E.; Mane, S.M.; Stephens, M.; Gilad, Y. RNA-seq: An assessment of technical reproducibility and comparison with gene expression arrays. Genome Res. 2008, 18, 1509-1517.

72. Benjamini, Y.; Hochberg, Y. Controlling the false discovery rate: A practical and powerful approach to multiple testing. J. R. Stat. Soc. B 1995, 57, 289-300.

(C) 2014 by the authors; licensee MDPI, Basel, Switzerland. This article is an open access article distributed under the terms and conditions of the Creative Commons Attribution license (http://creativecommons.org/licenses/by/3.0/). 